Xue-Guo Liu*, Wan-Yao Chen and Hui-Yan Pan

\title{
Crystal structure of $1,1^{\prime}$-(methylene)bis(pyridin-1- ium) bis(1,2-dicyanoethene-1,2-dithiolato-k $\left.{ }^{2} S: S\right)$ nickel(II), $\mathrm{C}_{42} \mathrm{H}_{30} \mathrm{~N}_{14} \mathrm{Ni}_{2} \mathrm{~S}_{8}$
}

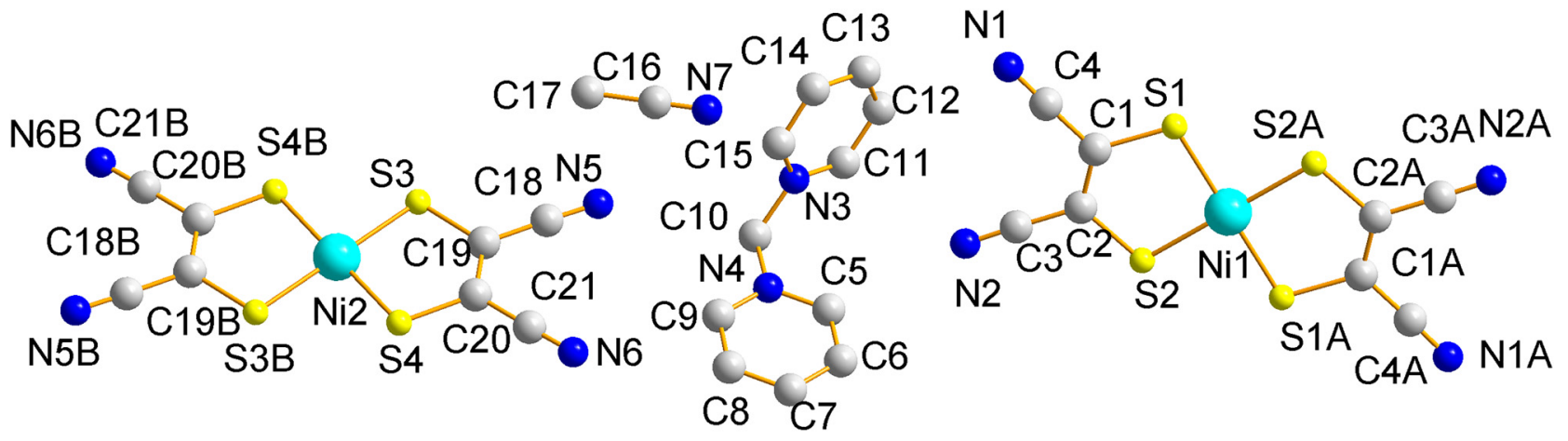

https://doi.org/10.1515/ncrs-2021-0434

Received November 9, 2021; accepted January 13, 2022;

published online February 2, 2022

\section{Abstract \\ $\mathrm{C}_{42} \mathrm{H}_{30} \mathrm{~N}_{14} \mathrm{Ni}_{2} \mathrm{~S}_{8}$, triclinic, $P \overline{1}$ (no. 2), $a=9.5083(17) \AA$, $b=10.744(2) \AA, c=12.188(2) \AA, \alpha=80.109(4)^{\circ}$, $\beta=84.876(4)^{\circ}, \gamma=88.758(3)^{\circ}, V=1221.7(4) \AA^{3}, Z=1$, $R_{\text {gtt }}(F)=0.0268, w R_{\text {ref }}\left(F^{2}\right)=0.0701, T=296.15 \mathrm{~K}$.}

CCDC no.: 2141881

Table 1 contains crystallographic data and Table 2 contains the list of the atoms including atomic coordinates and displacement parameters.

\section{Source of material} mercial sources and used without further purification. The starting materials disodium maleonitriledithiolate and 1, 1'-(methylene)bis(pyridin-1-ium)bromide were synthesized following the literature procedures $[3,4]$. An aqueous
All reagents and chemicals were purchased from com-

Table 1: Data collection and handling.

\begin{tabular}{ll}
\hline Crystal: & Red block \\
Size: & $0.23 \times 0.20 \times 0.18 \mathrm{~mm}$ \\
Wavelength: & Mo $K \alpha$ radiation $(0.71073 \AA$ A \\
$\mu:$ & $1.16 \mathrm{~mm}^{-1}$ \\
Diffractometer, scan mode: & $\varphi$ and $\omega$ \\
$\theta_{\text {max }}$, completeness: & $27.7^{\circ}, 99 \%$ \\
$N(h k l)_{\text {measured }}, N(h k l)_{\text {unique }}, R_{\text {int }}:$ & $14,613,5581,0.027$ \\
Criterion for $I_{\text {obs }}, N\left(h k l l_{\mathrm{gt}}:\right.$ & $I_{\text {obs }}>2 \sigma\left(I_{\text {obs }}\right), 4890$ \\
$N(\text { param })_{\text {refined }}:$ & 302 \\
Programs: & SHELX [1], Bruker [2] \\
\hline
\end{tabular}

solution (10 mL) of 1,1'-(methylene)bis(pyridin-1-ium) bromide $(0.0661 \mathrm{~g}, 0.2 \mathrm{mmol})$ was added slowly to an aqueous solution ( $15 \mathrm{~mL}$ ) of disodium maleonitriledithiolate $(0.0712 \mathrm{~g}, 0.4 \mathrm{mmol})$ and $\mathrm{NiCl}_{2} .6 \mathrm{H}_{2} \mathrm{O}(0.0463 \mathrm{~g}, 0.2 \mathrm{mmol})$, and the mixture was stirred at room temperature for several minutes. A red precipitate was filtered off, washed by water and dried under vacuum. The precipitate was dissolved in acetonitrile and dimethylformamide with ether diffusion. Fourteen days later red crystals were obtained.

\section{Experimental details}

*Corresponding author: Xue-Guo Liu, School of Biology and Chemical Engineering, Nanyang Institute of Technology, Nanyang 473004, China, E-mail: 512460415@qq.com. https://orcid.org/0000-00016331-9025

Wan-Yao Chen and Hui-Yan Pan, School of Biology and Chemical Engineering, Nanyang Institute of Technology, Nanyang 473004, China

Ә Open Access. ๑ 2022 Xue-Guo Liu et al., published by De Gruyter. (c) BY International License.
Absorption corrections were applied by using multi-scan program. The structure was refined based on $F^{2}$ with the SHELXL software package. Hydrogen atoms were treated as riding atoms. The $U_{\text {iso }}$ values of the hydrogen atoms were set to $1.2 U_{\text {eq }}(\mathrm{C})$. 
Table 2: Fractional atomic coordinates and isotropic or equivalent isotropic displacement parameters $\left(\AA^{2}\right)$.

\begin{tabular}{|c|c|c|c|c|}
\hline Atom & $x$ & $y$ & $z$ & $U_{\text {iso }}{ }^{*} / U_{\text {eq }}$ \\
\hline $\mathrm{C} 1$ & $0.42192(18)$ & 0.75001 (15) & 0.93099 (13) & 0.0370 (3) \\
\hline $\mathrm{C} 2$ & $0.56160(17)$ & $0.76129(15)$ & $0.89510(13)$ & $0.0360(3)$ \\
\hline C3 & 0.63737 (19) & $0.66774(17)$ & $0.84277(16)$ & $0.0446(4)$ \\
\hline $\mathrm{C} 4$ & $0.3417(2)$ & $0.64191(18)$ & $0.92029(16)$ & $0.0471(4)$ \\
\hline C5 & 0.88675 (17) & 0.33807 (16) & $0.80122(15)$ & 0.0385 (4) \\
\hline H5 & 0.839258 & 0.413493 & 0.778913 & $0.046^{*}$ \\
\hline C6 & $0.97260(18)$ & $0.32824(18)$ & $0.88625(16)$ & $0.0468(4)$ \\
\hline $\mathrm{H} 6$ & 0.983144 & 0.396523 & 0.922421 & $0.056^{*}$ \\
\hline $\mathrm{C} 7$ & $1.04376(18)$ & $0.2163(2)$ & $0.91831(16)$ & $0.0494(5)$ \\
\hline $\mathrm{H} 7$ & 1.102710 & 0.208706 & 0.976057 & $0.059^{*}$ \\
\hline C8 & $1.02670(18)$ & $0.11634(18)$ & $0.86416(16)$ & $0.0476(4)$ \\
\hline $\mathrm{H} 8$ & 1.074516 & 0.040672 & 0.884728 & $0.057^{\star}$ \\
\hline C9 & $0.93922(17)$ & $0.12861(16)$ & 0.78002 (15) & 0.0401 (4) \\
\hline H9 & 0.926818 & .060871 & 3592 & $0.048^{*}$ \\
\hline C10 & 0.78502 (17) & 0.25269 (17) & 0.65288 (13) & $0.0383(4)$ \\
\hline $\mathrm{H} 10 \mathrm{~A}$ & 0.829266 & 0.313223 & 0.591997 & $0.046^{*}$ \\
\hline 10B & 0.780618 & 0.172223 & 0.627467 & $0.046^{*}$ \\
\hline C11 & 0.60006 (19) & $0.41425(16)$ & $0.64345(16)$ & $0.0443(4)$ \\
\hline H11 & 0.662705 & 0.469753 & 0.597527 & $0.053^{*}$ \\
\hline $\mathrm{C} 12$ & $0.4644(2)$ & $0.45276(18)$ & $0.67121(18)$ & $0.0529(5)$ \\
\hline $\mathrm{H} 12$ & 0.434572 & .534344 & 0.644006 & $0.063^{*}$ \\
\hline C13 & $0.3736(2)$ & $0.3695(2)$ & 0.73947 (17) & $0.0528(5)$ \\
\hline H13 & 0.282155 & 0.394997 & 0.759577 & $0.063^{*}$ \\
\hline C14 & $0.41792(19)$ & $0.2488(2)$ & $0.77794(16)$ & $0.0542(5)$ \\
\hline H14 & 0.356604 & 0.191682 & 0.823429 & $0.065^{\star}$ \\
\hline C15 & $0.55263(17)$ & $0.21350(17)$ & $0.74883(14)$ & $0.0411(4)$ \\
\hline H15 & 0.583398 & 821 & 4577 & $0.049^{*}$ \\
\hline C16 & $0.5872(3)$ & $0.1532(2)$ & 0.40578 (19) & $.0627(6)$ \\
\hline C17 & $0.6025(3)$ & $0.0519(2)$ & 0.34180 (19) & $0.0719(6)$ \\
\hline H17A & 0.681454 & 0.068627 & 0.286663 & $0.108^{*}$ \\
\hline H17B & 0.518129 & .045836 & 0.305326 & $0.108^{*}$ \\
\hline $\mathrm{H} 17 \mathrm{C}$ & 0.618162 & -0.026201 & 0.390825 & $0.108^{*}$ \\
\hline C18 & $0.8358(2)$ & $-0.12958(18)$ & $0.57556(16)$ & 0.0477 (4) \\
\hline C19 & 0.90563 (17) & $-0.24751(15)$ & 0.56991 (14) & 0.0364 (3) \\
\hline $\mathrm{C} 20$ & $1.00398(17)$ & $-0.29073(15)$ & $0.64180(14)$ & $0.0366(3)$ \\
\hline $\mathrm{C} 21$ & $1.0363(2)$ & $-0.22038(18)$ & 0.72606 (17) & 0.0477 (4) \\
\hline N1 & $0.2746(2)$ & $0.55663(18)$ & $0.91448(18)$ & $0.0706(5)$ \\
\hline $\mathrm{N} 2$ & $0.70046(19)$ & 0.59504 (17) & $0.79991(17)$ & $0.0633(5)$ \\
\hline N3 & $0.64128(13)$ & $0.29584(12)$ & $0.68320(10)$ & $0.0313(3)$ \\
\hline N4 & $0.87062(13)$ & $0.23871(12)$ & $0.74947(11)$ & $0.0320(3)$ \\
\hline N5 & $0.7818(2)$ & $-0.03458(17)$ & $0.58174(18)$ & $0.0729(6)$ \\
\hline N6 & $1.0604(2)$ & $-0.16311(19)$ & $0.79275(18)$ & $0.0725(6)$ \\
\hline N7 & $0.5744(3)$ & $0.2312(2)$ & $0.4567(2)$ & $0.0927(7)$ \\
\hline $\mathrm{Ni1}$ & 0.500000 & 1.000000 & 1.000000 & $0.02856(7)$ \\
\hline $\mathrm{Ni} 2$ & 1.000000 & -0.500000 & 0.500000 & $0.03001(8)$ \\
\hline S1 & $0.33334(4)$ & $0.86928(4)$ & $0.98950(4)$ & $0.03823(10)$ \\
\hline S2 & $0.65479(4)$ & $0.89470(4)$ & $0.90973(3)$ & $0.03589(10)$ \\
\hline S3 & $0.86132(4)$ & $-0.33534(4)$ & $0.47093(4)$ & $0.03766(10)$ \\
\hline S4 & $1.08996(4)$ & $-0.43333(4)$ & $0.63615(4)$ & $0.03938(10)$ \\
\hline
\end{tabular}

\section{Comment}

Much effort has been devoted to the study of square-planar $\left[\mathrm{M}(\mathrm{mnt})_{2}\right]^{n-}$ complexes because of their structures and properties in conducting and magnetic materials, dyes, non-linear optics and catalysis [5-10]. Recently, maleonitriledithiolate complexes have been obtained by selecting different divalent cations [11-14]. These salts generally consist of $\left[\mathrm{M}(\mathrm{mnt})_{2}\right]^{n_{-}}(\mathrm{M}=\mathrm{Ni}(\mathrm{II}), \mathrm{Pd}(\mathrm{II}), \mathrm{Zn}(\mathrm{II})$, etc.) anions and cationic counterions. It is interesting to note that different cations or metals lead to significantly different stacking patterns. Herein, we choose 1,1'-(methylene)bis(pyridin-1-ium) as organic divalent cation to obtain the title salt. The title complex crystallizes in the triclinic space group $P \overline{1}$, with two half of $\left[\mathrm{Ni}(\mathrm{mnt})_{2}\right]^{2-}$, one 1,1'-(1,2-ethanediyl)bis(pyridin-1-ium) dication and one acetonitrile molecule in the asymmetric unit (see the figure). The presence of acetonitrile molecule is obviously different from the compounds previously synthesized [12-14]. Each Ni(II) ion is coordinated by four sulfur atoms, and exhibits a square-planar coordination geometry. The $\mathrm{Ni}-\mathrm{S}$ bond lengths and $\mathrm{S}-\mathrm{Ni}-\mathrm{S}$ bond angles are in agreement with reported values [12]. It should be noted the title structure possesses segregated stacking column. The C$\mathrm{H} \cdots \mathrm{N}$ interactions are observed between anions and cations [15]. The three-dimensional supramolecular structure is formed by such weak interactions.

Author contributions: All the authors have accepted responsibility for the entire content of this submitted manuscript and approved submission.

Research funding: This study was financially supported by Scientific and Technological Research Projects of Henan Province (182102311077).

Conflict of interest statement: The authors declare no conflicts of interest regarding this article.

\section{References}

1. Sheldrick G. M. Crystal structure refinement with SHELXL. Acta Crystallogr. 2015, C71, 3-8.

2. Bruker. SMART APEX-II CCD; Bruker AXS Inc.: Madison, WI, USA, 2006.

3. Simmons H. E., Blomstrom D. C., Vest R. D. Thiacyanocarbons. II. Chemistry of disodium dimercaptomaleonitrile. J. Am. Chem. Soc. 1962, 84, 4756-4771.

4. Musilek K., Komloova M., Zavadova V., Holas O., rabinova M., Pohanka M., Dohnal V., Nachon F., Dolezal M., Kuca K., Jung Y. S. Preparation and in vitro screening of symmetrical bispyridinium cholinesterase inhibitors bearing different connecting linkageinitial study for Myasthenia gravis implications. Bioorg. Med. Chem. Lett 2010, 20, 1763-1766.

5. Tanaka H., Okano Y., Kobayashi H., Suzuki W., Kobayashi A. A Three-dimensional synthetic metallic crystal composed of single-component molecules. Science 2001, 291, 285-287. 
6. Bigoli F., Chen C. T., Wu W. C., Deplano P., Mercuri M. L., Pellinghelli M. A., Pilia L., Pintus G., Serpe A., Trogu E. F. $\left[\mathrm{Ni}\left(\mathrm{R}_{2} \text { pipdt }\right)_{2}\right]\left(\mathrm{BF}_{4}\right)_{2}\left(\mathrm{R}_{2}\right.$ pipdt $=1$,4-disubstituted- piperazine-3,2dithione) as useful precursors of mixed-ligand dithiolenes of interest for non-linear optics. Chem. Commun. 2001, 2246-2247; https://doi.org/10.1039/b106064n.

7. Robertson N., Cronin L. Metal bis-1,2-dithiolene complexes in conducting or magnetic crystalline assemblies. Coord. Chem. Rev. 2002, 227, 93-127.

8. Duan H. B., Ren X. M., Meng Q. J. One-dimensional (1D) $\left[\mathrm{Ni}(\mathrm{mnt})_{2}\right]^{-}$based spin-Peierls-like complexes: structural, magnetic and transition properties. Coord. Chem. Rev. 2010, 254, 1509-1522.

9. Wang P. Crystal structure of $\mathrm{Mn}(\mathrm{II})\left(\mathrm{H}_{2} \mathrm{O}\right)(2,13$-dimethyl3,6,9,12,18-pentaazabicyclo[12.3.1]octadeca1(18),2,12,14,16-pentaene)-bis-(maleonitriledithiolate) nickel(II), $\mathrm{C}_{23} \mathrm{H}_{25} \mathrm{MnN}_{9} \mathrm{NiOS}_{4}$. Z. Kristallogr. N. Cryst. Struct. 2012, 227, 511-512.

10. Nishijo J., Uchida M., Enomoto M., Akita M. A chromium(III) bisacetylide complex containing a trans-diethyl-ethylenedithio- substituted tetrathiafulvalene (TTF) derivative: synthesis, crystal structures, and magnetic properties. Transit. Met. Chem. 2021, 46, 373-380.

11. Yan W. H., Ji E. Y., Shen M. L., Li Z. Y., Li X., Xu X. L. Two ion-pair complexes constructed by $\left[M(m n t)_{2}\right]^{n-}(M=N i, C o, m n t=$ maleonitriledithiolate): syntheses, characterization and thermal stability. Chin. J. Struct. Chem. 2015, 34, 306-312.

12. Yan W. H., Pan H. Y. Crystal structure of 1,4-bis(methylpyridinium benzene) bis(1,2-dicyanoethene-1,2-dithiolato- $\kappa^{2} S: S$ ) nickel(II), $\mathrm{C}_{26} \mathrm{H}_{18} \mathrm{~N}_{6} \mathrm{NiS}_{4}$. Z. Kristallogr. N. Cryst. Struct. 2021, 236, 107-108.

13. Yan W. H., Liu X. G., Shen M. L., Pan H. Y. Crystal structure of 1,1'(1,4-phenylenebis(methylene))bis(pyridin-1-ium) bis (1,2-dicyanoethene-1,2-dithiolato- $\kappa^{2} S: S$ ) zinc(II), $\mathrm{C}_{26} \mathrm{H}_{18} \mathrm{~N}_{6} \mathrm{ZnS}_{4}$. Z. Kristallogr. N. Cryst. Struct. 2021, 236, 533-535.

14. Liu X. G., Pan H. Y. Crystal structure of 1,1'-(1,3-phenylenebis (methylene))bis(pyridm)bis(1,2-dicyanoethene-1,2-dithiolato$\mathrm{K}^{2}$ S:S)palladium(II), $\mathrm{C}_{26} \mathrm{H}_{18} \mathrm{~N}_{6} \mathrm{PdS}_{4}$. Z. Kristallogr. N. Cryst. Struct. 2021, 236, 1247-1249.

15. Jeffrey G. A. Hydrogen-bonding: an update. Crystallogr. Rev. 2003, 9, 135-176. 